\title{
Attention Deficit Hyperactivity Disorder Symptoms Mediate Early-Onset Smoking
}

\author{
A.C. Huizink ${ }^{a}$ P.A.C. van Lier ${ }^{a}$ b A.A.M. Crijnen ${ }^{a}$ \\ a Erasmus Medical Center Rotterdam/Sophia Children's Hospital, Department of Child and Adolescent Psychiatry,

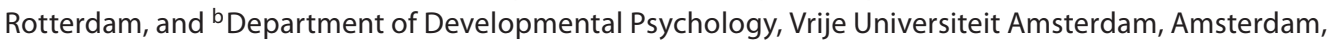 \\ The Netherlands
}

\section{Key Words}

Attention deficit hyperactivity disorder - Early-onset smoking $\cdot$ Mediating effect

\begin{abstract}
Background/Aims: Symptoms of attention deficit hyperactivity disorder (ADHD) have often been associated with early-onset smoking. We hypothesize that reductions in ADHD symptoms due to an intervention have a mediating effect on early-onset smoking. Methods: In a universal, school-based, randomized controlled intervention trial, we examined whether intervention-induced reductions in ADHD symptoms at age 9 mediated the reduced risk of tobacco use onset among these children at age 10 or 11 years. A sample of 477 first-grade boys and girls were randomly assigned to the Good Behavior Game intervention ( $n=263$ ), a 2-year (grades 2 and 3) universal classroom-based intervention aimed at reducing disruptive behavior problems, or to a control condition $(n=214)$. ADHD symptoms were assessed through teacher ratings. Early onset of tobacco use was assessed through self-report. Results: The intervention-induced reductions in ADHD symptoms fully mediated the distal effect of intervention on reductions in early-onset smoking. Conclusions: Our results showed that programs that target ADHD symptoms may protect children from early-onset smoking as well. Further research is needed to examine pathways from ADHD symptoms to tobacco use.
\end{abstract}

Copyright $\odot 2008$ S. Karger AG, Basel

KARGER
Fax +41 61306 1234
E-Mail karger@karger.ch
www.karger.com

(C) 2008 S. Karger AG, Basel

$1022-6877 / 09 / 0151-0001 \$ 26.00 / 0$

Accessible online at:

www.karger.com/ear

\section{Introduction}

Early adolescence is a critical developmental period of exposure to substances, including tobacco use [1]. Most tobacco users initiate smoking in adolescence, and tobacco is often the first drug used [2]. Various studies have shown that early-onset smoking predicts progression to daily and dependent patterns of tobacco use at later ages $[3,4]$. A recent review has suggested that adolescent tobacco use may be associated with a wide range of early adult social and health problems, including psychiatric disorders such as antisocial personality disorder, major depression and anxiety disorder, sleep problems, academic problems, crime, and early parenthood [5]. It is therefore of importance to obtain a better understanding of the processes that are involved in early-onset tobacco use. With this knowledge, effective interventions can be developed to prevent youngsters from starting to smoke, and in particular to prevent smoking onset at an early age.

Symptoms of attention deficit hyperactivity disorder (ADHD) have often been associated with early-onset smoking $[6,7]$. Most previous research on the association of ADHD with cigarette smoking has been conducted within samples of clinically referred youths. Indeed, ADHD is often related to smoking and other kinds of substance abuse. Clinical studies have shown that one third to one half of patients (adults and adolescents) seeking treatment for substance use disorders have a history of ADHD $[8,9]$. Several other recent studies have focused on community samples. For instance, it was found that clinically 
significant ADHD inattention symptoms predict 'ever having smoked' in 1,066 tenth-grade students [10]. In the same study sample it was found that adolescent 'ever smokers' with symptoms of ADHD had high novelty-seeking scores. Thus, adolescents with novelty seeking and symptoms of inattention and hyperactivity may have an increased vulnerability to experiment with smoking [11]. A study that included a very large nationally representative sample of adults likewise showed that self-reported ADHD symptoms were associated with adult smoking outcome, thereby providing further evidence of a link between ADHD symptoms and risk for tobacco use [7].

It is, however, unknown whether ADHD symptoms are actually causally related to tobacco use, or whether they simply coincide. To make claims about causation more probable, randomized controlled trials have been proposed because they provide the unique capacity to test for reciprocity between hypothesized risk variables and outcomes, while the randomization controls for possible sources of confounding $[12,13]$. There are some indications that ADHD symptoms may indeed cause smoking. For instance, the results of a preliminary study among college students using medication to control their ADHD symptoms indicate that lowering the level of $\mathrm{ADHD}$ symptoms may be important to protect against increased risk of tobacco use [14].

In the present prospective study we tested the link between ADHD symptoms and early-onset tobacco use. We specifically studied whether reductions in ADHD symptoms at age 9 , as a proximal outcome of a classroom-based intervention targeting disruptive behavior problems, mediated the reduced risk of tobacco use at age 10 or 11 years among children who received the intervention. In a previous study, we found that at age 7 , children who were prenatally exposed to maternal smoking had higher ADHD symptom scores. The Good Behavior Game (GBG) intervention did not affect the course of their ADHD symptoms and the probability of early-onset experimentation with smoking. This indicates that prenatally exposed children were not susceptible to a positive impact of the intervention [15]. Therefore, we adjusted for prenatal maternal smoking in the present study. Also, current parental smoking was adjusted for. Finally, it should be noted that some authors have suggested that the risk of developing substance use disorder, including smoking in adolescents with ADHD, is actually mediated through conduct disorder, since ADHD and conduct disorder often co-occur $[16,17]$. We therefore tested whether reductions in conduct symptoms could also mediate the effect of the intervention on early tobacco use.

\section{Methods}

\section{Participants}

In 1999, mainstream elementary schools with at least 2 firstgrade classes in the metropolitan areas of Rotterdam and Amsterdam, the Netherlands, were recruited to participate in a schoolbased preventive intervention study targeting disruptive behavior. The first 13 schools that responded positively to the invitation to participate were included [for more details, see 18]. The original target sample consisted of 794 first-grade children. Only the 722 children who moved on to second grade and the 22 children who repeated second grade, and who thus moved into the study cohort, were eligible for inclusion, resulting in a total sample of 744 children. All parents were approached to obtain written informed consent, and 666 parents (89.5\%) agreed to let their child participate in the study. At baseline, the mean age of these children was 6.9 years $(\mathrm{SD}=0.6)$, and $52 \%$ were male. During the summer break, when second-grade class compositions were known, classes within each school were randomly assigned to the intervention or control condition. Of the 31 classes in the 13 schools, 16 were assigned to the intervention condition and 15 to the control condition. Over the 2-year intervention period, 19 children moved from the control group into the intervention group. These children were treated as intervention children. In this period, 91 children dropped out of the study cohort because they failed to pass to the next grade or moved away from school. In addition, 1 school refused to participate after third grade $(n=55)$. During 2 followup measures, when children were 10 and 11 years of age, data for 43 children were missing because parents refused to let their child participate in these follow-up measures or because we were unable to locate them. Consequently, data were complete for 477 children (214 control group, 263 intervention children), yielding a response rate of $71.6 \%$ over a period of 6 years. Children who dropped out of the study during this phase were more likely to be female $\left[\chi^{2}(1, \mathrm{n}=677)=6.53, \mathrm{p}<0.05\right]$, of non-Caucasian ethnicity $\left[\chi^{2}(1, \mathrm{n}=677)=70.66, \mathrm{p}<0.01\right]$ and of low socioeconomic status $(\mathrm{SES})\left[\chi^{2}(1, \mathrm{n}=624)=31.57, \mathrm{p}<0.01\right]$. Figure 1 shows a flow chart in which the numbers of included children in each step of the study are presented. Fifty-four percent of the remaining 477 children were boys. Eighty-two percent of the children were Caucasian, $7 \%$ were of Moroccan, $7 \%$ were of Turkish and $4 \%$ of other ethnic background. Thirty percent of the households were of low SES, which resembles the percentage of low SES families in the general Dutch population [19].

\section{GBG Intervention}

The GBG is a classroom-based behavior management strategy that promotes prosocial behavior and aims to reduce disruptive behavior, including hyperactive and impulsive behavior. The GBG offers a well-structured task within the classroom, including clear behavioral rules, and provides systematic reward. In previous reports, the GBG has demonstrated to reduce symptoms of ADHD [20] and aggressive problems associated with high levels of ADHD symptoms [20,21]. In the present study, we will focus specifically on the reduction of ADHD symptoms after the GBG intervention. In the GBG, teachers discuss the necessity of formulating class rules and, with their students, select positively formulated class rules, which are accompanied by pictograms. After observing children on well-defined behaviors in the classroom, teachers assign children to 1 of the 3 or 4 teams. Teams include 


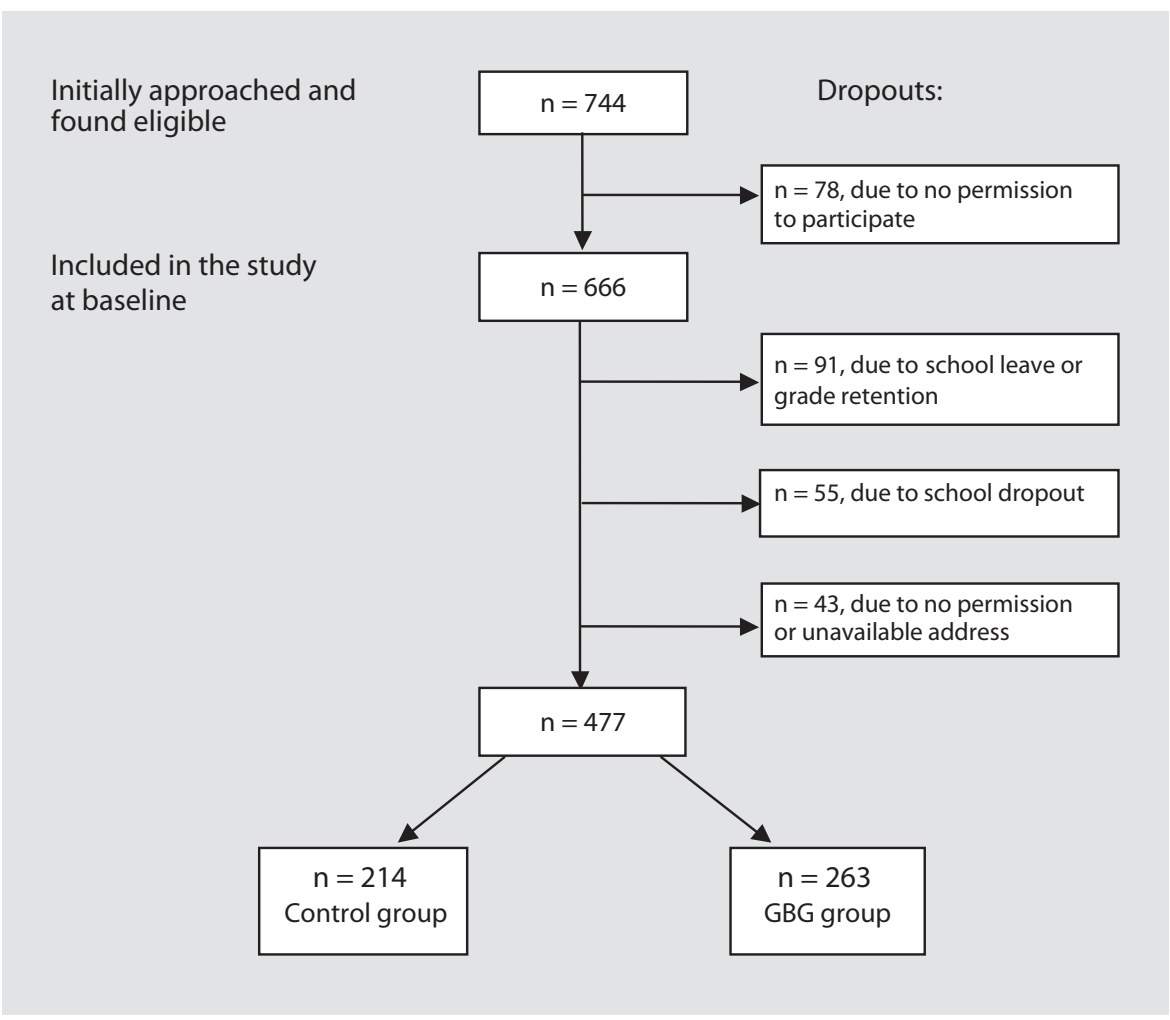

Fig. 1. Flow chart of the response of participants.

equal numbers of disruptive and nondisruptive children. Children are encouraged to manage their own and their teammates' behavior through a process of group reinforcement and through mutual self-interest. Each team receives a number of cards and teams are rewarded when at the end of a 15- to 60-min period at least 1 card remains. Teachers take a card when students violate 1 of the chosen rules. Students are always rewarded with compliments.

In the first intervention year, the GBG was implemented in 3 different stages. In the introduction phase, the GBG was played 3 times a week during $10 \mathrm{~min}$. In addition to the compliments, winning teams also received tangible rewards (stickers) directly after each game. In the expansion phase, teachers were encouraged to expand the duration of the GBG (up to three 1-hour sessions per week), expand the settings in which the GBG was played and expand the behaviors targeted by the GBG. Rewards were delayed until the end of the week and, later, until the end of the month. This phase lasted until early spring of the school year. In the generalization phase, emphasis was on promoting prosocial behavior outside GBG moments by explaining children that the rules used during the GBG were also applicable when the game was not in process. Children received compliments for appropriate behavior by their teachers. The GBG sessions were used as a booster. The same 3 phases were used in the second intervention year, but since the children were already familiar with the GBG, teachers swiftly moved to the expansion and generalization phase.

The GBG was implemented in second and third grade (age 7 and 8 years). Teachers received 2 afternoons of training prior to the intervention and 1 afternoon of instruction in midyear. Dur- ing the first intervention year, teachers were coached in their classroom during ten 60 -min classroom observations by welltrained advisors from the school advisory services.

External school advisors evaluated implementation fidelity based on the frequency of the intervention periods and the total amount of intervention time in hours per class. Of the 13 schools, 9 implemented the GBG program completely. Three schools implemented the introduction and expansion stage. Despite differences in implementation fidelity of the GBG, an intention-to-treat approach was used throughout the analyses. The GBG was initiated by the third author [22]. It was adapted for use in the Netherlands by the educational services $[23,24]$.

\section{Measures}

Assessment of Symptoms of ADHD

Teacher ratings of ADHD symptoms were determined over the last 2 months by means of the Teacher's Report Form (TRF)/6-18 [25], which contains a list of 120 items on which the child's behavior is rated on a 3-point scale. These ratings do not yield psychiatric diagnoses. In the present study, the 13 items resembling DSMIV symptoms of ADHD were used [26]. The total score of ADHD could range between 0 and 26. Items included information on inattention (for example, 'this child has difficulty with concentration') as well as on behavioral impulsivity and hyperactivity (for example, 'this child is impulsive', 'this child talks out of turn', 'this child finds it hard to sit still'). These 13 items were summed to a total ADHD baseline score, which was used as the indicator for the pre-, peri- and postintervention latent variables (time 1, 2 and 4) in the analyses model (see Statistical Analysis section; fig. 1). The 
TRF has been translated and validated for use in the Netherlands [27]. Cronbach's $\alpha$ ranged from 0.90 to 0.91 over the assessments.

At 2 assessment waves (time 3 and 4), teachers were interviewed with the Problem Behavior at School Interview (PBSI) [28]. The PBSI is a 32-item interview assessing disruptive and shy/ withdrawn behavior in children and was developed by two of our authors (AAMC, PACL). Teachers rated the child's behavior on a 5 -point Likert scale. The ADHD problem scale was used in this study. The ADHD scale consists of 8 items, 1 of which focuses on inattention, whereas the other items focus on impulsivity and hyperactivity. The total score could range between 0 and 32. Items included 'this child has difficulty with concentration' and 'this child is impulsive'. The interrater reliability, which reflected the correlation coefficients between 2 teachers who rated the same children, was $r=0.45$ ( $p<0.01)$. Cronbach's $\alpha$ was $>0.90$. The summed score served as the indicator for the time 3 and 4 latent ADHD variable (fig. 1). Thus, at time 4 , both the total ADHD scores of the PBSI and TRF served as indicators for the time 4 latent $\mathrm{ADHD}$ variable.

\section{Assessment of Conduct Problems}

Teacher-rated conduct problems were assessed at the same time points as ADHD symptoms through the TRF/6-18 and PBSI. The 11 items (TRF/6-18) and 9 items (PBSI) on conduct problems include 'this child is mean to others', 'this child fights', 'this child feels no guilt' and 'this child lies and cheats'. Cronbach's $\alpha$ was always $>0.90$ over the assessments.

\section{Assessment of Substance Use}

By means of self-report on the Substance Use Questionnaire [29], young adolescents filled in questions on tobacco, alcohol or other substance use at age 10 and 11, and on age of onset of using these substances at time 5 and 6 , when they were aged 10 and 11 years, respectively. Cigarette use was scored on a 7-point scale ranging from 0 (did not smoke at given age) to 7 (smoked more than 20 cigarettes per day). Because of our young sample, we focused on use versus nonuse ( $0=$ no tobacco use; $1=$ tobacco use, defined as 'smoking 1 cigarette or less a week' to 'smoking more than 20 cigarettes per day').

\section{Control Variables}

Women's use of cigarettes during pregnancy was assessed through the Substance Use during Pregnancy Interview [29]. When the children were 10 years old, trained interviewers contacted the mothers by telephone for information about the frequency of use and number of cigarettes they had smoked during pregnancy. Retrospective reporting of substance use during pregnancy may yield valid measures, whereas women may underreport substance use if asked during pregnancy because of stigma associated with substance use at that time. Especially when pregnancy has passed and there is no discernible major adverse effect on the child, the mother may be forthright in her reporting [30, 31]. In the present study, prenatal smoking was defined as a binary variable $(0=$ did not smoke during pregnancy; $1=$ smoked during pregnancy).

Current parental smoking was assessed during the same telephone interview when children were 10 years old. At the time of the assessment, parents were asked if they currently smoked. Current smoking was defined as a dichotomized variable: 0 ('no') if they did not currently smoke and 1 ('yes') if they did.

\section{Procedure}

Preintervention assessment of symptoms of ADHD (time 1, spring of grade 1 , age 7 years) was conducted with the TRF. Periand postintervention assessment of behavior also included the TRF at time 2 (spring of grade 2, age 8 years) and time 4 (early summer grade 4 , age 9 years). Teachers were sent 5 forms with preprinted names each week and were asked to complete 1 form per day. In this way, teachers filled out the TRF for each child in their class over a period of approximately 1 month for each assessment wave. In addition, teachers were interviewed with the Problem Behavior at School Interview [28] at time 3 (fall of grade 3) and time 4 (early summer grade 4 , age 9 years) for each of the classroom children. Postintervention assessment of substance use (time 5 and 6, age 10 and age 11, respectively) was conducted by means of self-report on the Substance Use Questionnaire [29], which was filled in by our subjects, supervised by research assistants, in the classroom. The children were told that their answers would be confidential and that they did not have to answer any of the questions if they did not want to. Teachers were not present in the classroom while the children were filling out the questionnaires.

\section{Statistical Analysis}

Figure 2 shows the mediation model we used for the present study. The objective of the model was to test whether reductions in the growth of ADHD symptoms over the ages of 7-9 years, due to the intervention, mediate the reductions in the probability of early-onset tobacco use [32]. The ADHD symptom scales of the TRF/6-18 and PBSI were used as indicators (TRF at time 1,2 and 4; PBSI at time 3 and 4) for the growth parameters.

To account for the missing-by-design data, the following procedure was used. First, a latent variable was considered for each of the 4 time points (time 1-4). Indicators for these latent variables were the total ADHD symptom scores derived from the TRF and PBSI at the given time points. Second, measurement invariance of $\mathrm{ADHD}$ symptoms across the 4 time points was approached as follows. (1) To put the 4 latent ADHD problem variables in the same metric at each of the 4 time points, the factor loading of the TRF on the latent variables at each time point was set by default at 1 , while the factor loading for PBSI was held equal across time. (2) The measurement intercepts were constrained to be equal across time for both the TRF and PBSI scores. The measurement model, which controlled for gender and GBG effects on the growth parameters, had a good fit to the data: $\chi^{2}(13, \mathrm{n}=477)=17.78$, $\mathrm{p}>0.05$; comparative fit index $=0.99$, Tucker-Lewis index $=0.98$, root mean squared error of approximation $=0.03$. To further ascertain measurement invariance between the TRF and PBSI, the factor loadings and measurement intercepts of the PBSI at time 3 and 4 were estimated (that is, not held equal across time). The model did not improve $\left[\chi^{2}(2, \mathrm{n}=477)=4.0, \mathrm{p}>0.05\right]$.

To test for mediation, the following procedure was used. First, to ascertain that our data fulfilled the requirements to test for mediation, the impact of intervention on the outcome (early-onset smoking) and growth of our hypothesized mediator (that is, impact on slope of ADHD symptoms) was tested, controlling for preintervention levels of ADHD symptoms (that is, intercept of ADHD symptoms) and male gender. In the second phase of the analyses, we specified the direct path from growth in ADHD symptoms to the outcome [32]. Sobel's test [33] was used to test for the significance of the indirect (mediation) path [34]. To con- 


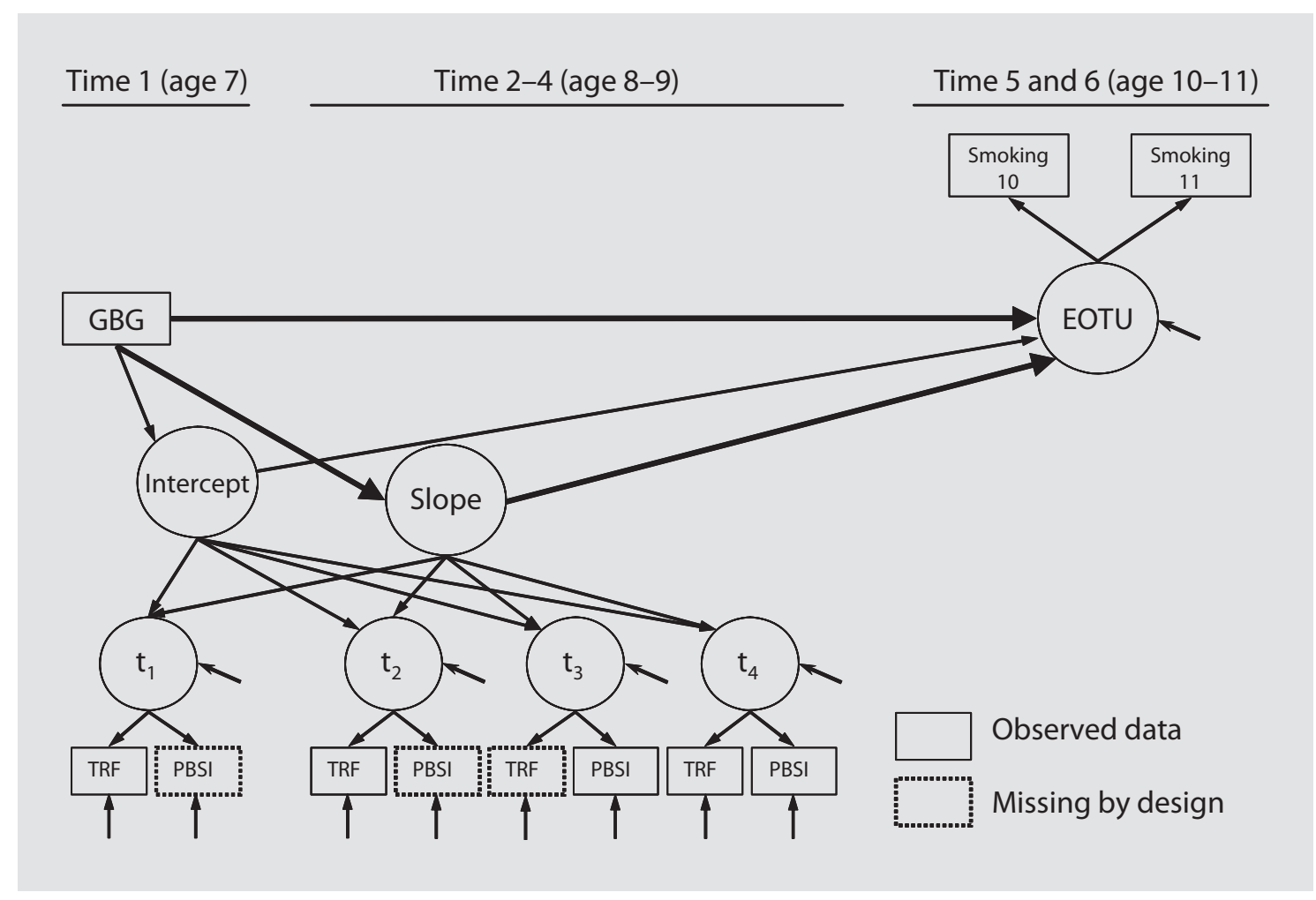

Fig. 2. Observed and latent variables to test for mediation of reduction in ADHD symptoms (direct effect of intervention) on the reductions in early-onset tobacco use (distal outcome of intervention). EOTU = Earlyonset tobacco use.

trol for possible nonindependence in the teacher ratings of ADHD symptoms (1 teacher filled out all questionnaires for his/her children in the classroom), parameter estimates were corrected for school level clustering, using a sandwich estimator. As such estimators affect the $\chi^{2}$ statistics and standard errors, means- and variance-adjusted test statistics [35] were used to test for significance of parameters. All models were fitted in Mplus 4.2 [36].

\section{Results}

\section{Descriptive Statistics}

A total of 61 children (12.8\%) reported tobacco use at age 10-11 years in the sample. However, children who received the GBG intervention had lower probability of early-onset tobacco use than control group children [GBG: 9.9\%; controls: $16.4 \% ; \chi^{2}(1, \mathrm{n}=477)=4.43, \mathrm{p}<$ 0.05]. The percentage of children from the control group that displayed early-onset tobacco use was in accordance with the general Dutch population [37]. Although specific medication use was not assessed in this sample, data on mental health service use was available. However, contact with a mental health institution was not related to intervention status (GBG vs. control): $\chi^{2}(1, \mathrm{n}=454)=3.4$, $p>0.05$. Other background characteristics and descriptive information on the intervention and control groups, such as gender, low SES and ADHD scores, are shown in table 1. There were no differences in gender or ADHD scores between the intervention and control groups. However, the intervention group contained more children of low SES.

\section{Intervention, ADHD Symptoms and Early-Onset Tobacco Use}

First, we specified a nonmediation model (fig. 3a). All parameter estimates were controlled for male gender. A significant effect of intervention status on the growth (slope) of ADHD symptoms $(\beta=-0.49, \mathrm{p}<0.01)$ indicated reduced growth rates of these problems in intervention children over the studied period. Holding the effect of intervention on the slope parameter equal to zero resulted in a significant deterioration of the model: $\chi^{2}$ $(1, \mathrm{n}=477)=14.68, \mathrm{p}<0.01$. Cohen's d was 0.27 , which reflects a medium effect size. As demonstrated previously, the reduction in early-onset tobacco use was also sig- 


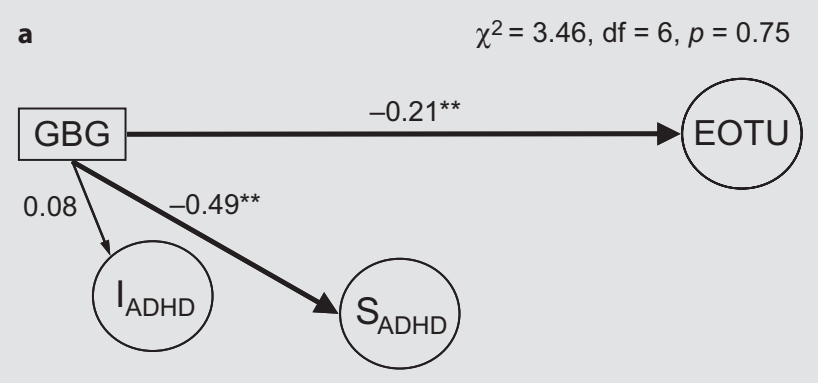

b

$\chi^{2}=1.94, \mathrm{df}=5, p=0.86$

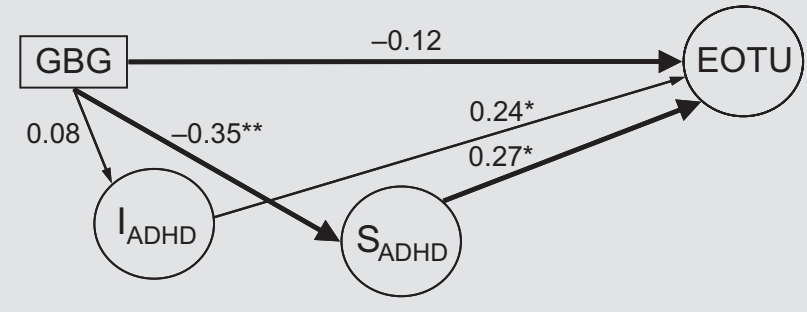

Fig. 3. Parameter estimates of the nonmediation model (a) and mediation model (b). EOTU = Early-onset tobacco use; $\mathrm{I}_{\mathrm{ADHD}}=$ Intercept of ADHD symptoms; $\mathrm{S}_{\mathrm{ADHD}}=$ slope of $\mathrm{ADHD}$ symptoms. ${ }^{*} \mathrm{p}<0.05 ;{ }^{* *} \mathrm{p}<0.01$.

Table 1. Background and descriptive information on the intervention and control group

\begin{tabular}{lcc}
\hline Variable & Intervention group & Control group \\
\hline Male gender & $55.9 \%(\mathrm{n}=147)$ & $51.9 \%(\mathrm{n}=111)$ \\
Low SES & $24.7 \%(\mathrm{n}=65)$ & $35.5 \%(\mathrm{n}=76)$ \\
ADHD time 1 (TRF) & $3.8(4.9)$ & $3.2(4.9)$ \\
ADHD time 2 (TRF) & $3.2(5.0)$ & $3.1(4.5)$ \\
ADHD time 3 (PBSI) & $17.1(7.1)$ & $18.2(7.9)$ \\
ADHD time 4 (PBSI) & $15.3(6.7)$ & $17.1(7.7)$ \\
ADHD time 4 (TRF) & $3.0(4.5)$ & $4.0(5.3)$ \\
\hline
\end{tabular}

Figures are presented as means with SD in parentheses, unless indicated otherwise.

nificant: $\beta=-0.21, p<0.01$. Again, holding the effect of intervention on tobacco use equal to zero significantly reduces the model fit: $\chi^{2}(1, \mathrm{n}=447)=10.69, \mathrm{p}<0.01$.

Next, to test for mediation, the direct paths from the growth parameters to early-onset tobacco use were specified. Both the intercept $(\beta=0.24, \mathrm{p}<0.05)$ and slope parameter $(\beta=0.27, \mathrm{p}<0.05)$ were significantly associ- ated with the outcome. Holding these parameters equal to zero (that is, the nonmediation model) significantly reduced the model fit: $\chi^{2}(1, \mathrm{n}=477)=5.96, \mathrm{p}<0.05$. The indirect path (GBG $>$ growth in ADHD symptoms $>$ early-onset tobacco use) was significant $(\beta=-0.10, p<0.05)$. Moreover, when specifying the indirect path, the direct path from intervention status to early-onset tobacco use was no longer significant $(\beta=-0.12, p>0.05)$, indicating that the reduction in ADHD symptoms completely mediated the intervention-induced reductions in early-onset tobacco use (fig. 3b).

To assess the robustness of this finding, we ran a number of tests. First, to test whether this effect was specific for the reduction in ADHD symptoms, we additionally tested the same models for reductions in symptoms of conduct problems. In the nonmediation model, we found a significant effect of the intervention status on the slope of conduct problems $(\beta=-0.08, p<0.05)$. However, the indirect path was not significant $(\beta=-0.01)$, indicating that reduction in conduct problems did not mediate the intervention-induced reductions in early-onset tobacco use.

We then tested whether 3 possible sources of confounding, namely prenatal exposure to maternal smoking, current parental smoking and low SES, influenced our findings. Both prenatal exposure to tobacco [38] and current parental smoking [39] have been associated with early-onset tobacco use in their offspring.

With respect to prenatal smoking, 83 mothers (17\%) reported having smoked during pregnancy. We first included this variable as a main effect in the nonmediation model predicting children's tobacco use at age 10-11 years, and in predicting the intercept and slope of symptoms of ADHD. In accordance with previous research, we found that the initial level (intercept) of ADHD symptoms was significantly associated with prenatal smoking $(\beta=0.23, p<0.05)$. No effect of prenatal smoking was found on the slope $(\beta=-0.04, p>0.05)$. This term was therefore deleted from the model. A trend toward significance was found for prenatal smoking on children's early-onset tobacco use $(\beta=0.27, p=0.06)$. The direct effect of intervention remained significant $(\beta=-0.40, p<0.05)$ when controlling for prenatal exposure to tobacco.

We then specified the mediation model. When taking into account the intervention-induced reductions in ADHD symptoms, the direct effect of intervention on early-onset smoking was no longer significant, confirming that even when controlling for prenatal smoking, intervention-induced reductions in ADHD symptoms mediated the reduced levels of early-onset tobacco use. Interestingly, the direct effect of prenatal exposure to 
tobacco on children's early-onset tobacco use was also rendered into insignificance $(\beta=0.16, p>0.05)$ after taking into account reductions in ADHD symptoms.

Current parental smoking was reported in $41 \%$ of the families. However, no effect of this factor was found on children's early-onset tobacco use $(\beta=0.19, \mathrm{p}>0.05)$. Similarly, no effect of current parental smoking was found on the growth parameters of ADHD symptoms from age 7 to 9 years.

Finally, the interaction effect of low SES was tested. We first included the interaction term between intervention status and low SES in predicting the slope of ADHD symptoms and the probability of experimentation with tobacco, to test whether GBG effectiveness depended upon SES. These interaction terms were not significant and were dropped from the analyses. We then controlled for the main effect of low SES. The indirect path from intervention status, via ADHD symptoms to tobacco use remained significant $(\beta=-0.08, p<0.05)$, suggesting that there was no influence of low SES on the results. The final model showed an effect size of 0.42 , which reflects a medium effect. The mean difference in the latent factor was 1.768 , with an SD of 4.23 .

\section{Discussion}

This study shows evidence for a mediating effect of ADHD symptoms on early-onset tobacco smoking in late childhood. Our results suggest that ADHD behavior is somehow involved in the pathway towards early-onset smoking. No such link was found for reductions in conduct problems and early-onset tobacco use. More specifically, reducing ADHD symptoms also seems to protect children from early-onset smoking. Although such evidence is not sufficient to come to causal conclusions, it provides some support for such a link [40]. Our findings were in accordance with previous reports among US young adolescents showing that by targeting behavioral outcomes such as ADHD, a distal preventive effect on early-onset smoking was also achieved $[41,42]$.

Our study focused on a young group of participants, at the verge of entering adolescence, which is a critical developmental period of exposure to tobacco and other (il)licit substances [1]. Early-onset smoking is related to an increased risk of regular smoking at later ages [43]. Furthermore, exposure to nicotine during adolescence, while brain development is still ongoing, may pose an additional risk for adverse outcomes. For instance, the prefrontal cortex is still developing and does not reach full maturation until early adulthood [44]. Likewise, transmission and receptor expression in the hippocampus of the neurotransmitter GABA-B is achieved in late adolescence [45]. Thus, early-onset smoking may hamper normal development and it is therefore of importance to examine which causal factors predict smoking. Our findings suggest that ADHD may be such a factor.

\section{Pathways from ADHD to Tobacco Use}

Several pathways through which symptoms of ADHD are linked with tobacco use have been suggested. One explanatory pathway may be that the inattention symptoms and behavioral impulsivity of children with ADHD may result in difficulties to understand and oversee the longterm consequences of smoking $[6,46]$.

Another explanation for the association between ADHD symptoms and high prevalence of smoking may be the self-medication theory. This theory implies that individuals with ADHD symptoms employ nicotine to enhance cognitive functioning [47]. Indeed, several studies have shown evidence for improvements on affective and cognitive measures, particularly on measures of sustained attention following nicotine administration in adults, which suggests a possibility of self-medication to account for greater prevalence of cigarette smoking among individuals with ADHD symptoms [48]. Indirect proof for the self-medication theory was provided by a study that showed that adolescents with ADHD, who were treated with pharmacotherapy, smoked less than their untreated counterparts over 2 years of high school [49].

A third pathway may be that children with symptoms of ADHD are more likely to get involved with antisocial peers [50], which puts them at a risk of early-onset smoking. Various studies have indeed shown that peer influences (affiliation with delinquent or antisocial peers, peers' drug use) [51] are associated with substance use. Moreover, the probability of such affiliation with deviant peers has been linked to childhood disruptive behavior problems [52]. In line with this pathway, Tarter et al. [53] provided a model in which early deficits in behavioral regulation are related to maladaptive social interactions, which in turn can increase the risk of having deviant and delinquent peers, and early-onset substance use.

\section{Limitations}

Several limitations have to be taken into account when interpreting our findings. First, a general population sample is representative, but is characterized by low prevalence rates of tobacco use, especially because of our young age groups, which may have influenced the results. 
In addition, we addressed early onset of smoking and therefore may have focused on predisposed children. It is of interest to follow our study population beyond the age of 11 . However, because of the importance to study correlates and predictors of tobacco use at an early age, our results contribute to the identification of adolescents at risk. Second, the possibility exists that our study omitted important mediating variables on our outcome [54]. For instance, other externalizing behavior such as childhood aggression and conduct disorder co-occur with symptoms of ADHD [55] and may be related to tobacco smoking as well. However, our results showed that reductions in conduct problems were not related to onset of tobacco use. This is in line with a recent population-based study, which showed that adjusting for conduct disorder did not change the significant association between ADHD and smoking [7]. In line with this, no strong association has been found as yet for childhood aggression and early-onset smoking. For instance, O'Callaghan et al. [38] have recently shown that the influence of prenatal and current maternal smoking had the strongest effect on early-onset smoking in a model that also included childhood aggression as predictor. In our study, we adjusted the analyses for the influence of prenatal maternal and current parental smoking. However, the small number of children that was exposed to especially prenatal smoking may have prevented us from drawing firm conclusions in this regard. Third, we used teacher ratings of ADHD symp- toms, which did not yield psychiatric diagnoses of ADHD. Moreover, in our study, we could not differentiate between various constructs within ADHD, such as inattention and hyperactivity. Therefore, we do not know which aspect of ADHD accounted for the effect on tobacco use. Our findings reflect that within a representative population sample, reductions in the number of ADHD symptoms have an influence on onset of tobacco use. Fourth, we have no biochemical confirmation on actual tobacco use and based our findings on self-reported use of at least 1 cigarette per week at age 10 or 11. Finally, our findings are limited to late childhood and preadolescence and to the onset of tobacco use. Our findings need to be extended to older adolescents and future studies may focus on the development from initiation of tobacco use to regular smoking.

\section{Implications}

Reducing ADHD symptoms also protects children from early-onset smoking. This finding is relevant for the development of prevention programs. Our findings suggest that these prevention programs should also focus on targeting behavioral symptoms, especially those related to ADHD, when aiming at preventing early-onset tobacco use. Future research should be directed at establishing which pathway, or combination of pathways, explains the link between ADHD and early smoking initiation.

\section{References}

1 Hopfer CJ, Crowley TJ, Hewitt, JK: Review of twin and adoption studies of adolescent substance use. J Am Acad Child Adolesc Psychiatry 2003;42:710-719.

$\checkmark 2$ Brown RA, Lewinsohn PM, Seeley JR, Wagner EF: Cigarette smoking, major depression, and other psychiatric disorders among adolescents. J Am Acad Child Adolesc Psychiatry 1996;35:1602-1610.

-3 Patton GC, Carlin JB, Coffey C, Wolfe R, Hibbert M, Bowes G: The course of early smoking: a population-based cohort study over three years. Addiction 1998;93:1251-1260.

-4 Costello EJ, Erkanli A, Federman E, Angold A: Development of psychiatric comorbidity with substance abuse in adolescents: effects of timing and sex. J Clin Child Psychol 1999; 28:298-311.

-5 Mathers CM, Toumbourou JW, Catalano RF, Williams J, Patton GC: Consequences of youth tobacco use: a review of prospective behavioural studies. Addiction 2006;101: 948-958.
6 Wilson JJ, Levin FR: Attention-deficit/hyperactivity disorder and early-onset substance use disorders. J Child Adolesc Psychopharmacol 2005;15:751-763.

-7 Kollins SH, McClernon FJ, Fuemmeler BF: Association between smoking and attention-deficit/hyperactivity disorder symptoms in a population-based sample of young adults. Arch Gen Psychiatry 2005;62:11421147.

8 Clure C, Brady KT, Saladin ME, Johnson D, Waid R, Rittenbury M: Attention-deficit/hyperactivity disorder and substance use: symptom pattern and drug choice. Am J Drug Alcohol Abuse 1999;25:441-448.

-9 Horner BR, Scheibe KE: Prevalence and implications of attention-deficit hyperactivity disorder among adolescents in treatment for substance abuse. J Am Acad Child Adolesc Psychiatry 1997;36:30-36.
10 Tercyak KP, Lerman C, Audrain J: Association of attention-deficit/hyperactivity disorder symptoms with levels of cigarette smoking in a community sample of adolescents. J Am Acad Child Adolesc Psychiatry 2002;41: 799-805.

-11 Tercyak KP, Audrain-McGovern J: Personality differences associated with smoking experimentation among adolescents with and without comorbid symptoms of ADHD. Subst Use Misuse 2003;38:1953-1970.

12 Kellam SG, Rebok GW: Building developmental and etiological theory through epidemiologically based preventive intervention trials; in Tremblay RE, McCord J (eds): Preventing Antisocial Behavior: Interventions from Birth through Adolescence. New York, Guilford Press, 1992, pp 162-195.

13 Rutter M, Pickles A, Murray R, Eaves L: Testing hypotheses on specific environmental causal effects on behavior. Psychol Bull 2001; 127:291-324 
14 Upadhyaya HP, Rose K, Wang W, O’Rourke K, Sullivan B, Deas D, Brady KT: Attentiondeficit/hyperactivity disorder, medication and treatment, and substance use patterns among adolescents and young adults. J Child Adolesc Psychopharmacol 2005;5:799-809.

15 Vuijk P, van Lier PA, Huizink AC, Verhulst FC, Crijnen AA: Prenatal smoking predicts non-responsiveness to an intervention targeting attention-deficit/hyperactivity symptoms in elementary schoolchildren. J Child Psychol Psychiatry 2006;47:891-901.

-16 Kandel DB, Johnson JG, Bird HR, Weissman MM, Goodman SH, Lahey BB, Regier DA, Schwab-Stone ME: Psychiatric comorbidity among adolescents with substance use disorders: findings from the MECA study. J Am Acad Child Adolesc Psychiatry 1999;38: 693-699.

17 Flory K, Lynam DR: The relation between attention deficit hyperactivity disorder and substance abuse: what role does conduct disorder play? Clin Child Fam Psychol Rev 2003;6:1-16.

- 18 Van Lier PAC, Verhulst FC, van der Ende J, Crijnen AAM: Classes of disruptive behavior in a sample of young elementary schoolchildren. J Child Psychol Psychiatry 2003;44: 377-387.

19 Statistics Netherlands: StatLine. Voorburg/ Heerlen, Netherlands Central Bureau of Statistics, 1999.

20 Van Lier PAC, Muthén BO, van der Sar RM, Crijnen AAM: Preventing disruptive behavior in elementary schoolchildren: impact of a universal, classroom based intervention. J Consult Clin Psychol 2004;72:467-478.

-21 Rebok GW, Hawkins WE, Krener P, Mayer LS, Kellam SG: Effect of concentration problems on the malleability of children's aggressive and shy behaviors. J Am Acad Child Adolesc Psychiatry 1996;35:193-203.

22 Crijnen AAM, Verhulst FC, Sturmans F: Sophia Foundation for Medical Research, Grant \#242. Department of Child and Adolescent Psychiatry. Sophia Children's Hospital/Erasmus Medical Center, Rotterdam, 1997.

23 Van der Sar AM, Goudswaard M: Teachers' Manual for the Good Behavior Game in Elementary Schools (in Dutch). Rotterdam, Pedologisch Instituut, 2001.

24 Van der Sar AM: Instructions for School Assistants and Teachers (in Dutch). Rotterdam, Pedologisch Instituut, 2002.

25 Achenbach TM: Manual for the Teacher's Report Form and 1991 Profile. Burlington, University of Vermont Department of Psychiatry, 1991.

26 Achenbach TM, Dumenci L, Rescorla LA: Ratings of Relations Between DSM-IV Diagnostic Categories and Items of the CBCL/618, TRF, and YSR. Burlington, University of Vermont Research Center for Children, Youth, and Families, 2001.
27 Verhulst FC, van der Ende J, Koot HM: Manual of the Teacher's Report Form (in Dutch). Rotterdam, Department of Child and Adolescent Psychiatry/Erasmus MC Rotterdam, 1997.

28 Crijnen AAM, van Lier PAC: Erasmus MC Problem Behavior at School Interview. Rotterdam, Department of Child and Adolescent Psychiatry, 2001.

29 Crijnen AAM, Vuijk P: Erasmus MC. Substance Use Questionnaire. Rotterdam, Department of Child and Adolescent Psychiatry, 2001

30 Ernhardt CB, Morrow-Tlucak M, Sokol RJ: Under-reporting of alcohol use in pregnancy. Alcohol Clin Exp Res 1988;12:506-511.

31 Jacobson SW, Jacobson JL, Sokol RJ, Martier SS, Ager JW, Kaplan MG: Maternal recall of alcohol, cocaine, and marijuana use during pregnancy. Neurotoxicol Teratol 1991;13: 535-540.

32 Cheong J, MacKinnon DP, Khoo S: Investigating the mediational processes using parallel process latent growth curve modeling. Struct Equat Model 2003;10:238-262.

33 Sobel ME: Asymptotic confidence intervals for indirect effects in structural equations models; in Leinhart S (ed): Sociological Methodology. San Francisco, Jossey-Bass, 1982, pp 290-312.

34 Baron RM, Kenny DA: The moderator-mediator variable distinction in social psychological research: conceptual, strategic, and statistical considerations. J Pers Soc Psychol 1986;51:1173-1182.

35 Asparouhov T, Muthén B: Robust chi square difference testing with mean and variance adjusted test statistics. Mplus Web Notes No. 10, 2006.

36 Muthén LK, Muthén BO: Mplus User’s Guide, ed 4. Los Angeles, Muthén \& Muthén, 2006.

37 Monshouwer K, Van Dorsselaer S, Gorter A: Youth and Risk Behavior: Main Findings from the Dutch National School Survey 2003 (in Dutch). Utrecht, Trimbos-Instituut, 2004.

38 O'Callaghan FV, O’Callaghan M, Najman JM, Williams GM, Bor W, Alati R: Prediction of adolescent smoking from family and social risk factors at 5 years, and maternal smoking in pregnancy and at 5 and 14 years. Addiction 2006;101:282-290.

39 den Exter Blokland EA, Engels RC, Hale WW 3rd, Meeus W, Willemsen MC: Lifetime parental smoking history and cessation and early adolescent smoking behavior. Prev Med 2004;38:359-368.

40 Rutter M, et al: Testing hypotheses on specific environmental causal effects on behavior. Psychol Bull 2001;127:291-324.

41 Furr-Holden CD, Ialongo NS, Anthony JC, Petras H, Kellam SG: Developmentally inspired drug prevention: middle school outcomes in a school-based randomized prevention trial. Drug Alcohol Depend 2004;73: 149-158.
42 Storr CL, Ialongo NS, Kellam SG, Anthony JC: A randomized controlled trial of two primary school intervention strategies to prevent early onset tobacco smoking. Drug Alcohol Depend 2002;66:51-60.

43 Robinson ML, Berlin I, Moolchan ET: Tobacco smoking trajectory and associated ethnic differences among adolescent smokers seeking cessation treatment. J Adolesc Health 2004;35:217-224.

44 Giedd JN: Structural magnetic resonance imaging of the adolescent brain. Ann NY Acad Sci 2004; 1021:77-85.

45 Luján R, Shigemoto R, Lopez-Bendito G: Glutamate and GABA receptor signalling in the developing brain. Neuroscience 2005; 130:567-580

46 Brown TE: Attention-Deficit Disorders and Comorbidity in Children, Adolescents and Adults. Washington DC, American Psychiatric Press, 2000

47 Dinn WM, Aycicegi A, Harris CL: Cigarette smoking in a student sample: neurocognitive and clinical correlates. Addict Behav 2004 29:107-126.

48 Poltavski DV, Petros T: Effects of transdermal nicotine on attention in adult non-smokers with and without attentional deficits. Physiol Behav 2006;87:614-624.

49 Whalen CK, Jamner LD, Henker B, Gehricke JG, King PS: Is there a link between adolescent cigarette smoking and pharmacotherapy for ADHD? Psychol Addict Behav 2003; 17:332-335

50 Laird RD, Jordan KY, Dodge KA, Pettit GS, Bates JE: Peer rejection in childhood, involvement with antisocial peers in early adolescence, and the development of externalizing behavior problems. Dev Psychopathol 2001;13:337-354

51 Von Sydow K, Lieb R, Pfister H, Hofler M, Wittchen HU: What predicts incident use of cannabis and progression to abuse and dependence? A 4-year prospective examination of risk factors in a community sample of adolescents and young adults. Drug Alcohol Depend 2002;68:49-64.

52 Deater-Deckard K: Annotation: recent research examining the role of peer relationships in the development of psychopathology. J Child Psychol Psychiatry 2001;42: 565-579.

-53 Tarter R, Vanyukov M, Giancola P, Dawes M, Blackson T, Mezzich A, Clark DB: Etiology of early age onset substance use disorder: a maturational perspective. Dev Psychopathol 1999;11:657-683.

54 Howe GW, Reiss D, Yuh J: Can prevention trials test theories of etiology? Dev Psychopathol 2002;14:673-694.

55 Connor DF, Edwards G, Fletcher KE, Baird J, Barkley RA, Steingard RJ: Correlates of comorbid psychopathology in children with ADHD. J Am Acad Child Adolesc Psychiatry 2003;42:193-200. 